\title{
Mechanical response to swift ion irradiation-induced nano-tracks in silica
}

\author{
Ángel R. Páramo, F. Sordo, D. Garoz, O. Peña-Rodríguez, A. Prada, J. Olivares, M.L. Crespillo, \\ J.M. Perlado, A. Rivera ${ }^{a}$
}

Ion irradiation on dielectric materials produces several processes, such ionization and defect formation followed by a decay governed by thermal processes such as heat diffusion and atomic rearrangement. Finally in the irradiated region the mechanical properties are altered, strain and stress fields appear, a densification takes places and other properties such as the refractive index are affected.

In order to simulate the mechanical response of silica to swift ion irradiation we use a methodology based on molecular dynamics (MD) and finite element methods (FEM). We use information from MD to obtain the local densification generated by an incoming swift ion. Finally we calculate the densification in the ion track using FEM. This method provides information on the strain and stress field along the material as a function of ion irradiation fluence.

For this work an experimental campaign using Br ions from 5 to $50 \mathrm{MeV}$ has been done at CMAM accelerator (Madrid). We measured the refractive index and we observe that for high fluences the refractive index decreases. The effect of the strain field on the density could explain the decrease in the refractive index. We check this hypothesis using our methodology coupling MD and FEM.

\section{Introduction}

Heavy ion irradiation in silica with energies $>0.1 \mathrm{MeV} / \mathrm{amu}$ is the origin of several effects of technological relevance. On one hand, it leads to material modification, usually, with detrimental results (as in Nuclear fusion facilities [1]) and on the other hand, it can be used to optimize or tailor the physical properties, e.g., as in waveguide fabrication $[2,3]$.

In this type of irradiation, energy is transferred from the projectile mainly to electrons that subsequently produce significant impact ionization. As a result, in a nm-sized region around the ion trajectory a high electronic excitation density is achieved. Due to several processes the stored energy is transferred to the lattice in ps timescales. The consequence of the sudden energy enhancement is strong atom disorder, bond breaking and temperature raise followed by a fast atom rearrangement and cool-down. Permanent effects appear in $\mathrm{nm}$-sized tracks with modified density, stoichiometry, composition and defect level that seriously affect the physical properties.

Effects of ion irradiation in silica have been already studied in different works [4-7]. In these works the track morphology, stresses and strains under irradiation are explained. However no study shows the variation of the refractive index with the ion fluence. Therefore we study how the optical properties of silica are modified upon ion irradiation. We try to correlate the observed changes in the refractive index to variations in the stress/strain fields originated by the irradiation. The conclusion is that the overall mechanical properties affect marginally to the refractive index variation.

\section{Experiments}

$\mathrm{Br}$ ion irradiation (5-40 MeV) of silica was done at CMAM [8]. In situ optical reflectance measurements were carried during the irradiations as depicted in Fig. 1. The measurements show that the reflectance increases with fluence in all cases. It is straightforward to obtain the near-surface refractive index variation during 


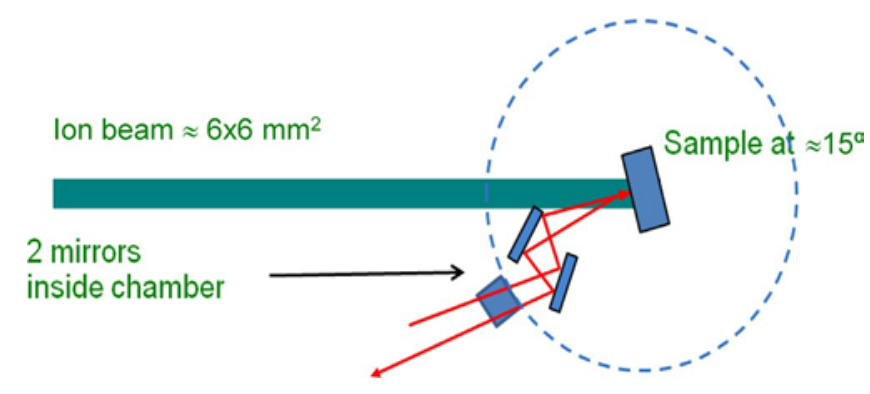

Fig. 1. Schematic representation of the setup for in situ reflectance measurements. A sample can be irradiated with ions and at the same time illuminated with white light (arrows). The reflected light is detected by a spectrometer.

irradiation from the reflectance (25 MeV Br irradiation in Fig. 2). Br ions produce tracks with higher density than the virgin material in the silica surface. Initially, when tracks do not overlap the refractive index variation is linear. However, when track overlapping becomes important the refractive index tends to saturate. An interesting effect occurs for fluences $>10^{13} \mathrm{~cm}^{-2}$, because, unexpectedly, the refractive index decreases.

We have compared the experimental results with a parametric model. In the parametric model we relate the refractive index with the near-surface density using the Lorentz-Lorenz formula [9],

$$
\begin{aligned}
& \frac{2}{\underline{p}} \text { p 8:0324 } \\
& 8: 0324-q
\end{aligned}
$$

where $q$ is the density and $n$ is the refractive index. In order to calculate the refractive index in a heterogeneous medium (tracks and substrate), the Bruggeman expression is used,

$f_{A}^{A} \frac{\underline{n}^{2}{ }_{n_{A}^{2}} \mathrm{p} 2 n}{2} \mathrm{p} f_{B} \frac{n_{B}^{2}-n^{2}}{n^{8} \mathrm{p} 2 n^{2}} 1 / 40 ;$

where $f$ is the volume fraction in the near-surface region and subscripts $A$ and $B$ refer to tracks and substrate, respectively. The volume fraction of tracks of radius $r$ is related to the fluence ( $)$ through a Poisson distribution,

$f_{A} 1 / 41-e^{-/ \cdot p \cdot r^{2}}:$

In Fig. 2 we compare the experimental results to the parametric model. Good agreement is achieved at fluences $/<10^{13} \mathrm{~cm}^{-2}$ for track radius $r=3 \mathrm{~nm}$ and a density variation, $\mathrm{D} q / q=3.5 \%$. However at high fluences, $/>10^{13} \mathrm{~cm}^{-2}$ the experimental refractive index decreases whereas the model predicts saturation. Therefore, the model cannot explain correctly the observed behaviour at high fluencies when track overlapping occurs and a continuous layer is formed.

The question is why the refractive index decreases when the continuous layer is formed. In the next section we address this question and study the effect of strain relaxation when the continuous layer is formed on the refractive index.

\section{Simulations}

We used a finite element method (FEM) using Ansys APDL to study the strain field and relaxation at high fluences. The irradiation effect is taken into account in the FEM model through density variation at different fluences. By means of MD simulations (details published elsewhere) we estimate the track density for different electronic stopping power $\left(S_{\mathrm{e}}\right)$. In order to model the thermal evolution of the track region, we have used atomistic molecular dynamics with the Feuston-Garofalini interatomic potential [10]. The simulation boxes have $30 \times 30 \times 14 \mathrm{~nm}^{3}$ in order to simulate

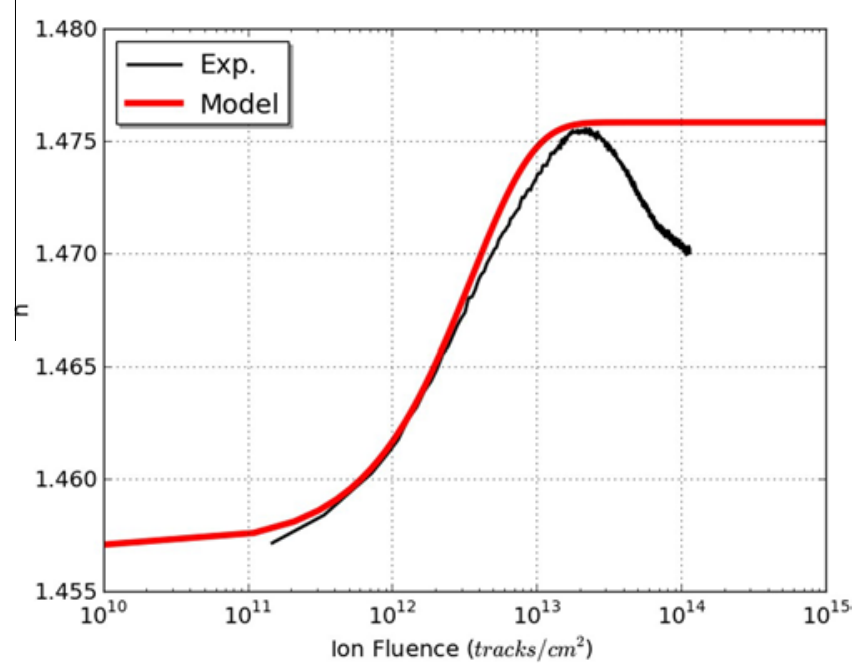

Fig. 2. Variation of refractive index in silica as a function of fluence for $25 \mathrm{MeV} \mathrm{Br}$ irradiation. For comparison, results from the parametric models with $\mathrm{D} q / q=3.5 \%$ and $r=3 \mathrm{~nm}$ are shown

$>8 \times 10^{5}$ atoms in a time domain that exceeds $100 \mathrm{ps}$. The simulation code was MDCASK [11] run in 256-512 cores at CESVIMAMAGERIT. Ion irradiation in the simulation box is characterised by an energy deposition $\left(\mathrm{S}_{\mathrm{e}}\right)$ in a hot cylinder. The thermal evolution is studied and the final state (see Fig. 3 for $\mathrm{S}_{\mathrm{e}}=6 \mathrm{keV} / \mathrm{nm}$ ) shows the effects of ion irradiation in silica.

From the MD simulation for different $S_{\mathrm{e}}$ and the $\mathrm{S}_{\mathrm{e}}$ profile along the ion track depth, we can reconstruct the track shape. In Fig. $4 \mathrm{a}$ the density variation of an isolated track is shown as a function of depth and axial coordinates in a 2D colour map. As the fluence increases tracks overlap, see Fig. 4b. Finally, a continuous layer is formed, Fig. 4c. According to the MD simulations, $25 \mathrm{MeV} \mathrm{Br}$ ion irradiation ( $S_{\mathrm{e}}$ rv $6 \mathrm{keV} / \mathrm{nm}$ at the surface) leads to the formation of tracks with a radius at the surface, $r \mathrm{rv} 3 \mathrm{~nm}$ and a density increase $\mathrm{D} q / q$ rv $3 \%$. This type of tracks agree with the parametric model used to reproduce the experimental results in Fig. 2 $(r=3 \mathrm{~nm}, \mathrm{D} q / q=3.5 \%)$.

The track shape shown in Fig. 4 is used as an input for the FEM model to obtain the strain and stress fields in the $25 \mathrm{MeV} \mathrm{Br}$ ion irradiated silica for any fluence. Isolated tracks do not substantially

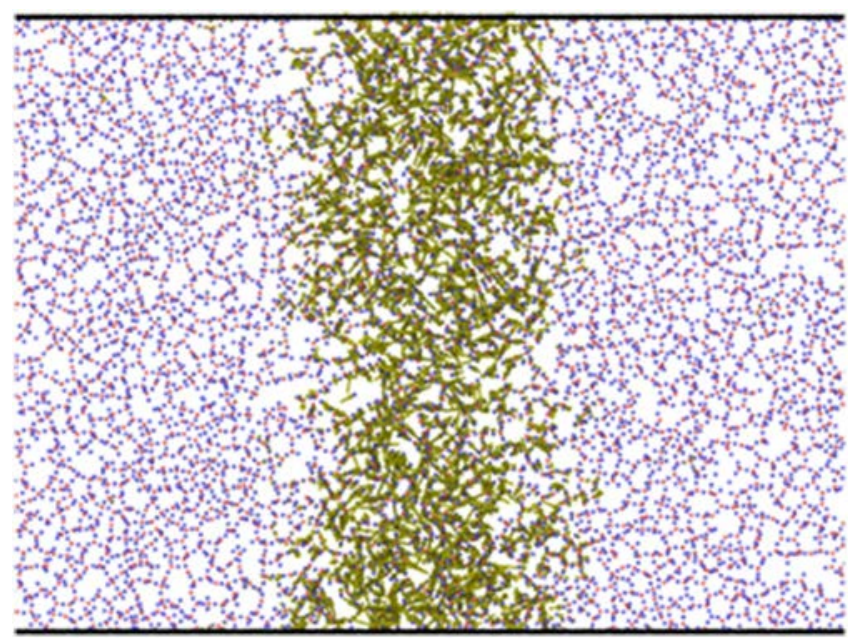

Fig. 3. Densified Track after irradiation with an ion of $6 \mathrm{keV} / \mathrm{nm}$. In the track the green arrows shows the displacement vector for the atoms, the average displacement towards the track centre leads to densification of the track. 

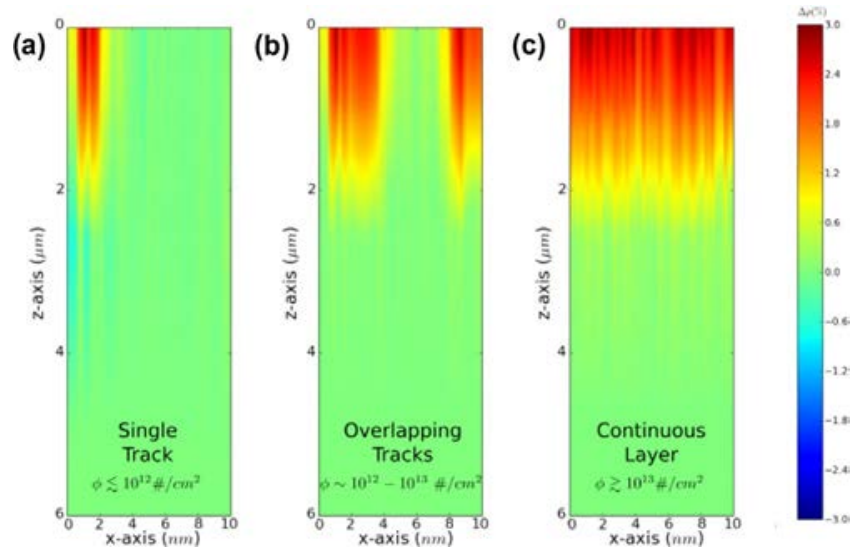

Fig. 4. Density variation in $25 \mathrm{MeV} \mathrm{Br}$-irradiated silica at different fluences (つ) (a) Isolated tracks $\left(/<10^{12} \mathrm{~cm}^{-2}\right)$. (b) Track overlapping $\left(/ \mathrm{rv} 10^{12}-10^{13} \mathrm{~cm}^{-2}\right)$. (c) Continuous layer $\left(/>10^{13} \mathrm{~cm}^{-2}\right)$. Note that depth and axial scales are different.

affect the surface. On the other hand, when tracks overlap and a continuous layer is formed, the irradiated surface cannot be flat any longer. It becomes curved to accommodate stresses resulting in a pronounced dip of several nanometres.

Regarding the stresses, if we apply an elastic model, stresses of hundreds of $\mathrm{MPa}$ appear upon track formation near the irradiated surface. These stresses are far above the yield stress for silica (48 MPa). Therefore, the surface enters the plastic regime and cracks may appear. In this regime, a plastic model is necessary to describe the material response to track accumulation. From the resulting strain field we can evaluate the density variation in the irradiated surface as

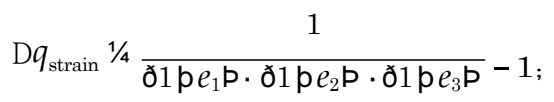

where $e_{1}, e_{2}, e_{3}$ are the principal strains.

In order to correlate the observed variation in the refractive index of silica under irradiation with the mechanical response of the material, we must make use of the expected changes in the near-surface density that in turn are responsible for the changes in the refractive index. Tracks with higher density than the unirradiated medium produce an overall density increase as they are formed. Thus, the density increases asymptotically to the value of the continuous layer formed upon track overlap (Fig. 5). In

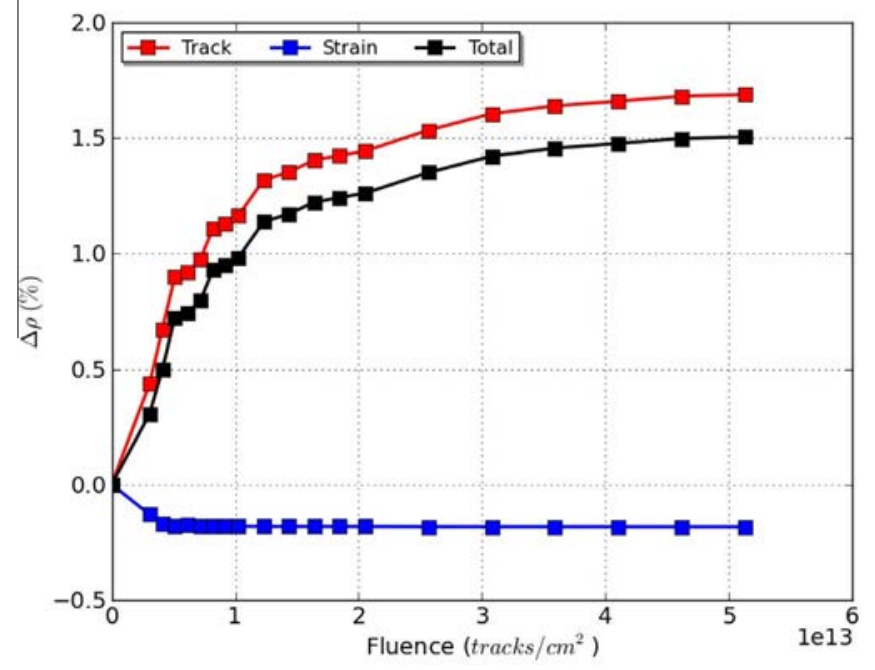

Fig. 5. Density variation due to the track and to the strain field for irradiation with $25 \mathrm{MeV} \mathrm{Br}$ ions. addition, the strain field introduces a negative correction to the total density according to (4) because the compressed tracks are under tensile stress by the surrounding medium. When plasticization of the surface occurs the surface strain does not change anymore with increasing fluence. Then, as observed in Fig. 5, the negative density correction due to the strain field saturates. The density correction due to the strain field turns out to be in any case much smaller in magnitude than the density enhancement due to track accumulation. Therefore, although stress relaxation occurs due to plasticization it cannot explain the observed decrease of the refractive index at high fluences.

\section{Conclusions}

In this work we have studied the variation of the refractive index of silica under irradiation. The results show a decrease in the refractive index when the tracks begin to overlap. This effect cannot be easily explained. We have studied the density variation due to track accumulation and overall strain relaxation and relate it to the evolution of the refractive index.

Using results from molecular dynamics simulations we reconstruct the track shape and use it as an input for a finite element model able to follow the mechanical response of silica with high density tracks. The model, taking into account plasticization, makes realistic predictions of surface variation and strain/stress fields.

We have focused on the possibility of a density decrease due to the strain relaxation at high fluences. In fact, the effect occurs but the density decrease is significantly smaller than the density increase due to track accumulation. We conclude that the strain relaxation is not responsible for the decrease in the refractive index at high fluencies.

Once this a priori obvious assumption is discarded, we are working on an alternative explanation to the effect based on reflectance changes due to enhanced rugosity.

\section{Acknowledgements}

The authors acknowledge the computer resources and technical assistance provided by CESVIMA and funding by MINECOSpain through projects AIC-A-2011-0718, MAT-2012-38541, and MAT2011-28379-C03-02.

\section{References}

[1] D. Garoz, R. González-Arrabal, R. Juárez, J. Álvarez, J. Sanz, J.M. Perlado, A. Rivera, Silica final lens performance in laser fusion facilities: HiPER and LIFE, Nucl. Fusion 53 (2013) 013010

[2] J. Manzano, J. Olivares, F. Agulló-López, M.L. Crespillo, A. Moroño, E. Hodgson, Optical waveguides obtained by swift-ion irradiation on silica (a-SiO2), Nucl. Instr. Meth. Phys. Res. B 268 (2010) 3147-3150.

[3] J. Olivares, A. García-Navarro, G. García, A. Méndez, F. Agulló-López, A. GarcíaCabañes, M. Carrascosa, O. Caballero, Nonlinear optical waveguides generated in lithium niobate by swift-ion rradiation at ultralow fluences, Opt. Lett. 32 (17) (2007) 2587-2589.

[4] B. Afra, M.D. Rodriguez, C. Trautmann, O.H. Pakarinen, F. Djurabekova, K. Nordlund, T. Bierschenk, R. Giulian, M.C. Ridgway, G. Rizza, N. Kirby, M. Toulemonde, P. Kluth, SAXS investigations of the morphology of swift heavy ion tracks in a-quartz, J. Phys. Condens. Matter Inst. Phys. J. 25 (2013) 045006.

[5] M.L. Brongersma, E. Snoeks, T. Van Dillen, A. Polman, Origin of MeV ion irradiation-induced stress changes in SiO2, J. Appl. Phys. 88 (2000) 59-64.

[6] S. Klaumünzer, Ion tracks in quartz and vitreous silica, Nucl. Instr. Meth. Phys. Res. B 225 (2004) 136-153.

[7] A. Benyagoub, S. Klaumünzer, M. Toulemonde, Radiation-induced compaction and plastic flow of vitreous silica, Nucl. Instr. Meth. Phys. Res. B 146 (1998) 449-454.

[8] Centro de Microanálisis de Materiales <www.cmam.uam.es>

[9] K. Vedam, P. Limsuwan, Piezo- and elasto-optic properties of liquids under high pressure. II. Refractive index vs density, J. Chem. Phys. 69 (1978) 4772.

[10] B.P. Feuston, S.H. Garofalini, Empirical three-body potential for vitreous silica, J. Chem. Phys. 89 (1988) 5818.

[11] Anon, The ASCI MDCASK Benchmark Code. 